\title{
Assessment of Nitazoxanide Loaded on Silver nanoparticles Efficacy on Treatment of Murine Model of Chronic Toxoplasmosis
}

\author{
Mohammed H. Saleh ${ }^{a}$, Ibrahim M. Nagaty ${ }^{a}$, Rabab S. Zalat ${ }^{b}$, kawkab A. Ahmed ${ }^{c}$, Doaa I. \\ Yaseen $^{\text {a }}$, Reda M. Abdelhameed ${ }^{\text {d }}$, Shereen M. Kishik ${ }^{\text {a }}$
}

a Department of Parasitology, Faculty of Medicine, Benha University, Egypt. ${ }^{\mathrm{b}}$ Department of Parasitology, Theodore Bilharz Research Institute, Egypt. c Department of Pathology, Faculty of Veterinary Medicine, Cairo University , Egypt. ${ }^{\mathrm{d}}$ Department of Applied Organic Chemistry, Chemical Industries Research Division, National Research Centre, Egypt.

Correspondence to: Doaa I. Yaseen, Department of Parasitology, Faculty of Medicine, Benha University, Egypt.

Email:

yaseendoaa7@gmail.com

Received: 18 January 2021

Accepted: 22 January 2021

\begin{abstract}
:
Background: Silver nanoparticles (AgNPs) can be used as vehicles to transport drug molecules to target zones and there by improve their therapeutical efficacy. Aim: The aim of the present work was to assess the effect of nitazoxanide loaded on silver nanoparticles (AgNps) in treatment of chronic toxoplasmosis in mice. Materials and methods: Mice were infected with $T$. gondii Me49 non-virulent strain and divided into 2 subgroups according to the time of starting treatment, and each subgroup sub divided into 7 groups. GI: Non infected- non- treated, GII: Infected non-treated mice, GIII: Infected and treated by spiramycin, GIV: Infected and treated by nitazoxanide, GVa: Infected and treated by nitazoxanide loaded on silver nanoparticles, GVb: Infected and treated by nanosilver only, GVI: infected and treated by combination of nitazoxanide and spiramycin, GVII: infected and treated by combination of nitazoxanide loaded on silver nanoparticles and spiramycin. Effect of treatment was assessed by counting the number of $T$. gondii brain cysts, histopathology and scanning electron microscopy. Results: Nitazoxanide loaded on AgNps significantly reduced the number of T. gondii brain tissue cysts and improved the inflammatory and pathological changes in brain tissue sections in treated groups as compared to the other groups. It also affects the normal architecture and size of tissue cysts in brain. Conclusion: Nitazoxanide loaded
\end{abstract} on AgNps proved their effectiveness against the experimental chronic Toxoplasma infection.

Keywords: Toxoplasmosis, Nitazoxanide (NTZ), Silver Nanoparticles (AgNps), Phosphate puffer solution (PBS). 


\section{Introduction}

Over one billion people worldwide are predicted to harbor Toxoplasma infection frequently with unknown lifelong health consequences. Currently available therapies are ineffective for persistent chronic disease and congenital toxoplasmosis or have severe side effects which may result in life threatening complications so, there is an urgent need for safe and effective therapies to treat this disease [1].

Nitazoxanide, 2-acetyloxy-N-(5-nitro-2thiazolyl) benzamide is a new nitrothiazole benzamide compound notable for its activity in treating both intestinal protozoal and helminthic infections [2]. Advances in nanomedicine allow the use of nanotechnology in the management of infectious diseases. Most common are the silver nanoparticles, which have antimicrobial and anti-inflammatory activity [3].

The present study aimed to evaluate the efficacy of nitazoxanide loaded on silver nanoparticles on treatment of chronic toxoplasmosis as an attempt to overcome shortcomings of other traditional drugs.

\section{Materials and methods}

A prospective experimental study was conducted on 80 laboratory mice selected from the animal house of Theodore Bilharz
Research Institute. The study conducted in Theodore Bilharz Research Institute, and Benha Faculty of Medicine from May 2018 to October 2018. The experiment was carried out according to the internationally valid guide lines and the research protocol was approved by Research Ethics Committee, Faculty of Medicine, Benha University, Egypt.

\section{Parasite and animals}

Me49 non-virulent strain of $T$. gondii (kindly obtained from Theodore Bilharz Research Institute, Egypt) was regularly maintained by repeated oral inoculation through oesophageal tube of Swiss albino mouse with $100 \mu \mathrm{l}$ of brain homogenate of previously infected mice containing approximately $1 \times 10^{2}$ tissue cysts per mouse every 8 weeks [4]. A total of 80 laboratorybred male Swiss albino mice 8-10 weeksold, approximately 20-25gram.

\section{Drugs}

\section{Nitazoxanide drug}

Nitazoxanide, available as suspension forms (100mg/5ml) manufactured by AlAndalous for Pharmaceutical Industries was used. Each 5ml suspension contains: Active Ingredients: Nitazoxanide 100mg 


\section{Nitazoxanide loaded on silver nanoparticles (AgNps)}

I .Synthesis of the silver metal nanoparticles (Ag MNPs):

Silver metal nanoparticles were prepared using nonhydrolytic sol gel methods according to the literature with some slightly modifications [5].

II. Preparation of Nitazoxanide loaded on silver nanoparticles:

For the coupling of the nitazoxanide to the Ag NPs nanoparticles, $2.5 \mathrm{mg}$ nitazoxanide was dissolved in $10 \mathrm{~mL}$ chloroform then 10 mg $A g$ NPs nanoparticles was added to the medium, the mixture was then incubated for 24 hours under stirring. The mixture of different concentrations of nitazoxanide $(0$ $14 \mathrm{mg} / \mathrm{L}$ ) was added to $10 \mathrm{mg} A g ~ N P s$ nanoparticles in presence of $10 \mathrm{~mL}$ chloroform and the mixture was stirred at r.t. on a $30 \mathrm{rpm}$ shaker for 24 hours. After that the solutions at that point were centrifuged at $3000 \mathrm{rpm}$ at r.t. for $15 \mathrm{~min}$. For purification, the coming about supernatants was disposed of; and $20 \mathrm{~mL}$ of chloroform was included to each tube for resuspension. Along these lines, the re-suspended particles were centrifuged at $6000 \mathrm{rpm}$ at r.t. for $20 \mathrm{~min}$. The supernatants were once more disposed off, and the strong were isolated and dried in r.t [6].

\section{Spiramycin drug}

Spiramycin 3 M.I.U was used in a tablet form. It was provided by (Pharaonia
Pharmaceuticals - analytical standard code: J01FA02). Spiramycin tablets were smashed and used as powder. Then the powder was dissolved in $15 \mathrm{ml}$ water and given orally to mice using oesophageal tube.

\section{Experimental Design}

Mice were classified into 2subgroups, 40mice in each subgroup. Subgroup infected and started treatment 2 weeks post infection and sacrificed after 3 weeks from the start of treatment and subgroup infected and started treatment 6 weeks post infection and sacrificed after 3 weeks from the start of treatment. Further classified into 7 groups each (5mice) except group V (10mice in each subgroup).GI: Non -infected, nontreated GII: Infected non-treated mice. GIII: Infected and treated by spiramycin at a dose $200 \mathrm{mg} / \mathrm{kg}$ for 2 weeks [7]. GIV: Infected and treated by nitazoxanide at a dose 200mg/kg for7days [8]. GVa: Infected and treated by nitazoxanide loaded on silver nanoparticles at a dose $200 \mathrm{mg} / \mathrm{kg}$ for 7 days. GVb: Infected and treated by nanosilver only at a dose $0.72 \mathrm{mg} / \mathrm{kg}(18 \mu \mathrm{g} / 100 \mathrm{ml}$ PBS $)$ for 7 days [9]. GVI: Infected and treated by combination of nitazoxanide at a dose $100 \mathrm{mg} / \mathrm{kg}$ and spiramycin at a dose $100 \mathrm{mg} / \mathrm{kg}$. GVII: Infected and treated by combination of nitazoxanide loaded on silver nanoparticles at a dose $100 \mathrm{mg} / \mathrm{kg}$ and spiramycin at a dose $100 \mathrm{mg} / \mathrm{kg}$. 


\section{Evaluation of drug efficacy}

\section{Counting the $T$. gondii tissue cysts number in brain}

Part of each brain tissue was weighted (0.01gm) and homogenized after that dissolved in $1 \mathrm{ml}$ saline. Twenty microliters of this fluid were spread on a glass slide. After that, the slides were placed in room temperature to dry completely then fixed using methanol. Fixed air dried films in absolute methanol were stained using Giemsa stain diluted in 1:20 ratio with water for 10 minutes then washed with tap water. The slides were examined by using light microscope $(\times 40)$ for counting tissue cysts [10]. The brain tissue cysts were then estimated per $10 \mathrm{mg}$.

\section{Histopathological evaluation}

Specimens from brain tissues of each mouse were fixed separately in $10 \%$ neutral buffered formalin, dehydrated in different concentrations of alcohol, cleared with xylol, and embedded in paraffin blocks. Tissue sections of $5 \mathrm{~mm}$ thickness were stained with haematoxylin and eosin (H \& E)[11].

\section{Scanning electron microscopy}

- Parts of the homogenized brain were rinsed three times in PBS, pH 7.2 and placed in $2.5 \%$ glutaraldehyde with $3 \%$ sucrose in 0.1 M sodium cacodylate buffer, $\mathrm{pH}$ 7.2. They were post fixed for 1 hour in the dark with $1 \%$ Osmium tetroxide, $0.8 \%$ potassium ferrocyanide and $\mathrm{mM} \mathrm{CaCl} 2$ in 0.1 M cacodylate buffer, $\mathrm{pH}$ 7.2. Samples were then dehydrated in graded ethanol and embedded in PolyBed. Ultra-thin sections were stained with uranyl acetate and lead citrate viewed with a scanning electron microscope[12].

Statistical analysiCollected data were coded and introduced to a PC using the Statistical Package for Social Science (SPSS) for windows version 11.0. Data were represented as the mean \pm standard deviation (SD) $(n=10)$. The analysis of variance (ANOVA) procedure was used to clarify statistically significant differences between the studied groups. Post hoc test (Bonferroni) for pairwise group comparison was used to assess inter group difference each 2 groups.

Values were considered statistically significant when $P<0.05$. The infection reduction rate was assessed using the formula: (Mean value of the infected untreated group - mean value of infected treated group) X 100 / Mean value of infected untreated group [13].

\section{Results}

\section{Silver nanoparticles characterization}

The transmission electron micrograph of silver nanoparticles showed that, the average particle size is found as $20 \mathrm{~nm}$. The 
particles are well separated from the neighbouring nanoparticles. The particles are spherical in shape (Fig.1).

\section{Parasitological results}

A. Subgroup of mice infected and started treatment 2 weeks post infection and sacrificed after 3 weeks from the start of treatment:

A significant statistical difference when comparing between (GII) and all other groups that indicates an observable efficacy of all tested drug regimens (table 1).

- A significant statistical difference when comparing between (GIII) and all other groups except (GIV). Its mean cyst count is higher than mean cyst count of group (GIV) (table 1). A significant statistical difference when comparing (GIV) and all other groups except (GIII) and (GVI). Its mean cyst count is lower than (GIII) but higher than (GVI) showing better effect of spiramycin and nitazoxanide combination (table 1). A significant statistical difference when comparing between $(\mathrm{GVa})$ and that of all other groups except (GVI). Its mean cyst count is lower than (GVI) (table 1).

A significant statistical difference when comparing between $(\mathrm{GVb})$ and that of all other groups. Its mean cyst count is higher than all groups except (GII) (table 1).

A significant statistical difference when comparing between (GVI) and that of all other groups except (GIII) and (GVa). Its mean cyst count is lower than (GIII) but higher than $(\mathrm{GVa})$. A significant statistical difference when comparing between (GVII) and all other groups. Its mean cyst count is lower than that of all groups. $p<0.05$ : Significant, $\mathrm{p}<0.001$ : Highly Significant, p>0.05:non Significant.

B. Subgroup of mice infected and started treatment 6 weeks post infection and sacrificed after 3 weeks from the start of treatment:

A significant statistical difference when comparing between (GII) and all other groups that indicates an observable efficacy of all tested drug regimens (table 2).

A significant statistical difference was found when comparing between (GIII) and all other groups except (GIV). Its mean cyst count is higher than mean cyst count of (GIV) (table 2). A significant statistical difference was found when comparing (GIV) and all other groups except (GIII) and (GVI).Its mean cyst count is lower than (GIII) but higher than (GVI) showing better effect of spiramycin and nitazoxanide combination ( table 2). A significant statistical difference was found when comparing between ( $\mathrm{GVa}$ ) and that of all other groups except (GVI). Its mean cyst count is lower than (GVI) (table 2).

A significant statistical difference was reported when comparing between $(\mathrm{GVb})$ and that of all other groups, Its mean cyst 

count is higher than all other groups except (GII) (table 2).

A significant statistical difference was detcted when comparing between (GVI) and that of all other groups except (GIII) and (GVa). Its mean cyst count is lower than (GIII) but higher than (GVa) (table 2). A significant statistical difference when was found when comparing between (GVII) and all other groups. (GVII) achieved the best result. Its mean cyst count is lower than that of all other groups (table 2).

\section{Histopathological study}

Histopathological examination of brain sections from GII (infected non treated group) showed severe inflammatory reaction exhibited by massive necrosis and pyknosis of neurons associated with neuronophagia, moderate cellular vaculation and edema (Fig.2).

While sections of brain from GVa treated with nitazoxanide loaded silver nanoparticles (2ws post infection) revealed moderate inflammatory changes described as intracellular vaculation and edema, proliferation of glia cells moderate degenerated neurons and neuronophagia of degenerated neurons (Fig. 3). However, brain of mice from GVII treated with nitazoxanide loaded silver nanoparticles and spiramycin (2ws post infection) showed necrosis and pyknosis of neurons associated with neuronophagia, perivascular cuffing with mononuclear cells, severe intracellular vacuolation and edema (Fig. 4). Marked improvement occur in brain sections from GVa treated with nitazoxanide loaded silver nanoparticles (6ws pot infection) which showed regression in inflammatory reaction and neuropathic changes noticed by mild intracellular vacuolation and edema associated with proliferation of glia cells (Fig. 5). Examined sections from group VII treated with nitazoxanide loaded silver nanoparticles and spiramycin(6ws post infection) showed neuronophagia of necrotic neurons, mild to moderate inflammatory reaction in the form of perivascular cuffing with mononuclear cells, mild intracellular vacuolation and edema (Fig.6)

\section{Scanning electron microscope}

Brain samples from groups of mice started treatment 6weeks post- infection: GII (infected control group), GIV(treated with nitazoxanide) and GVII (treated with nitazoxanide loaded silver nanoparticles and spiramycin). In the infected control group, T. gondii tissue cysts appeared rounded with normal diameter $20 \mu \mathrm{m}$. They were surrounded by well-defined intact cyst wall with smooth surface. There were no projections or dimples on surface (Fig.7). In GIV treated with nitazoxanide, $T$. gondii tissue cyst lost normal architecture and 
shape, reduced in size and its surface showed dimples, projections and formation of large hole (Fig.8). The best effect on $T$. gondii brain tissue cyst was noticed in GVII treated with combination of nitazoxanide loaded silver nanoparticles and spiramycin which showed shrinkage in their size, loss of normal contour in addition to formation of dimples (Fig.9).

Table (1): Comparison between the mean brain cyst count, starting 2 weeks post infection.

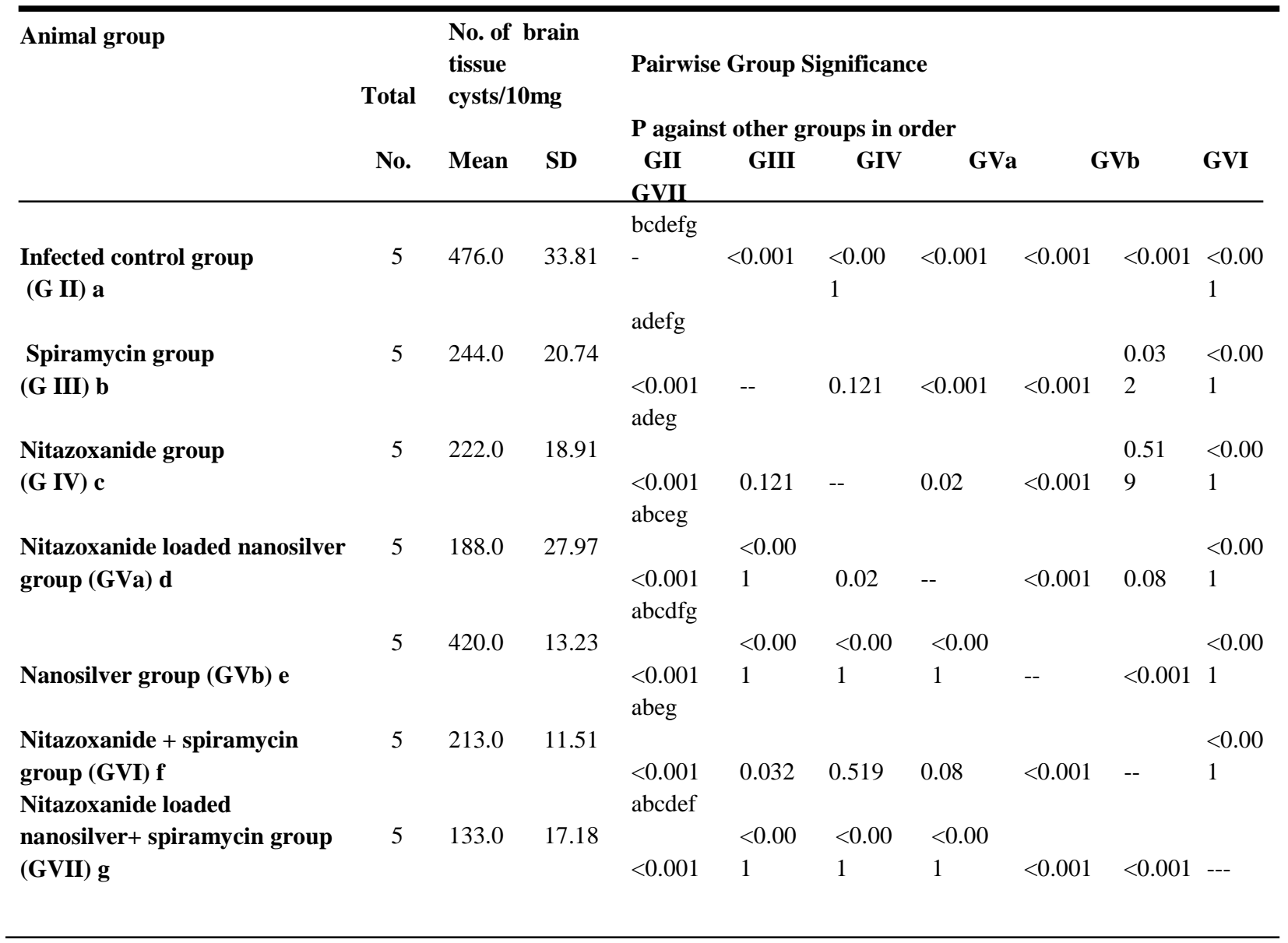


Table (2): Comparison between the mean brain cyst count, starting 6weeks post infection.

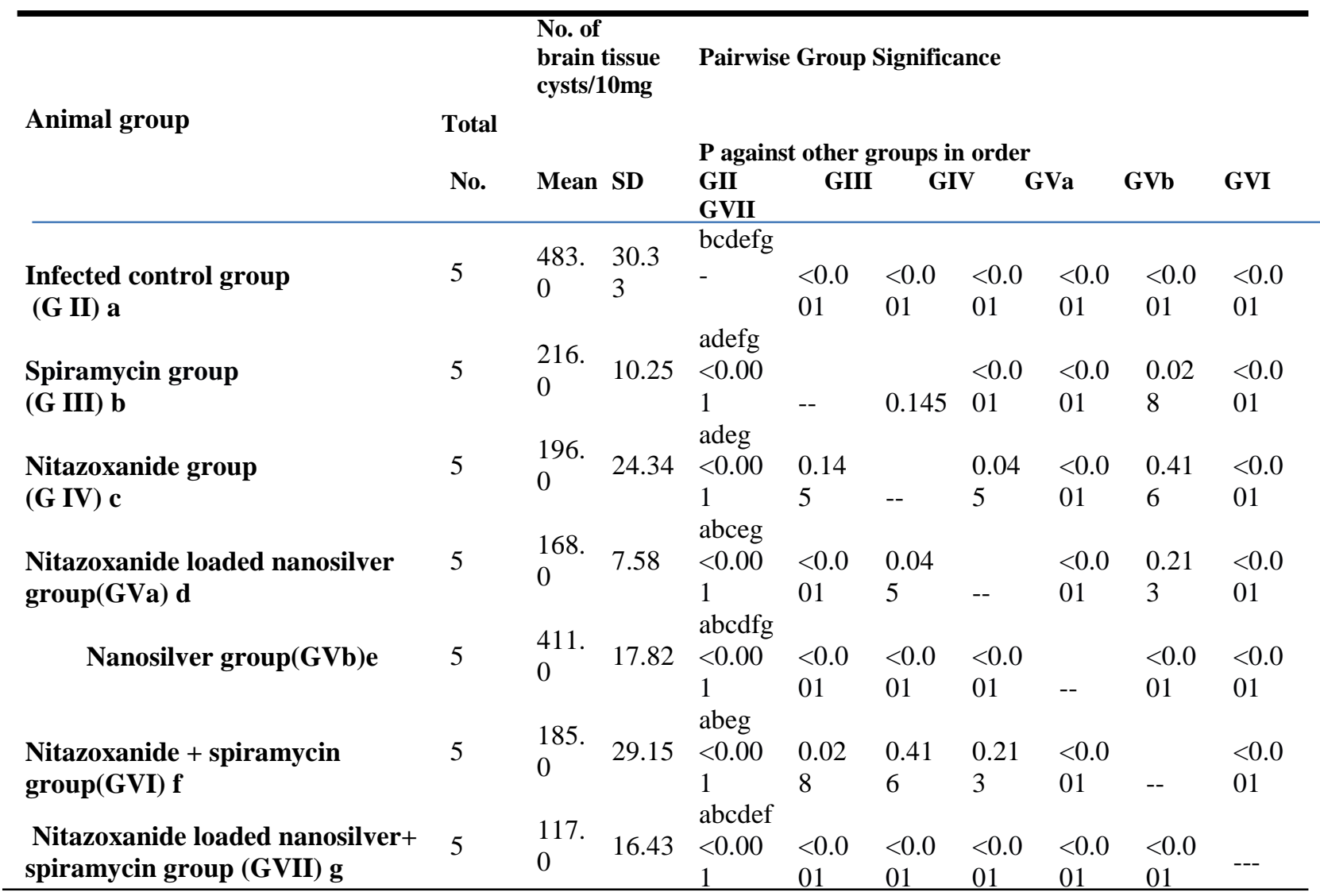

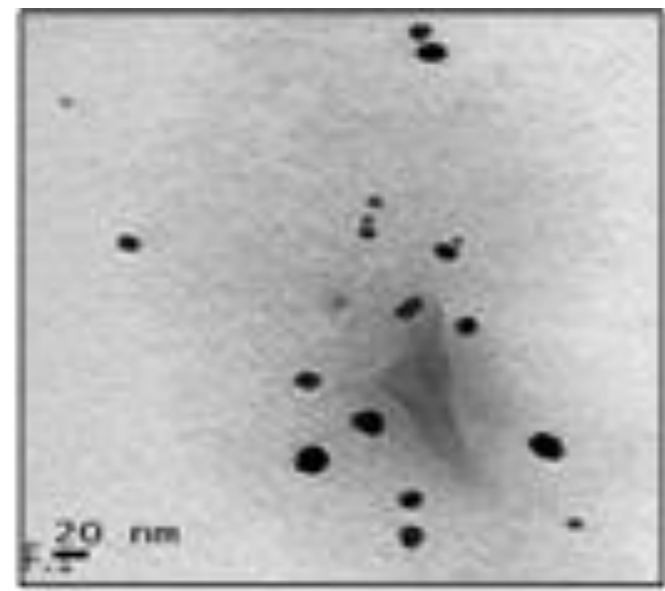

Figure1:Silver nanoparticles by TEM

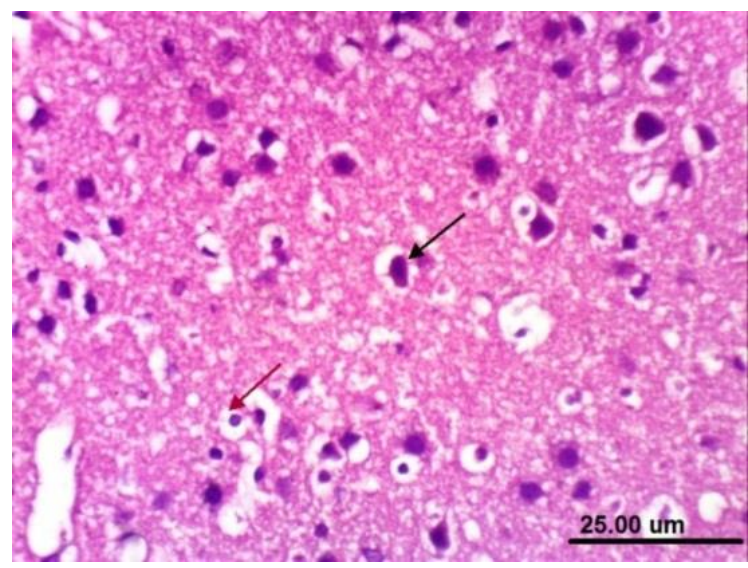

Figure 2: Brain of mice from group II showing massive necrosis and pyknosis of neurons (black arrow) as well as cellular vacuolation and edema (red arrow) Toxoplasma cysts embedded in tissues (white arrow) (H \& E, scale bar 25um). 


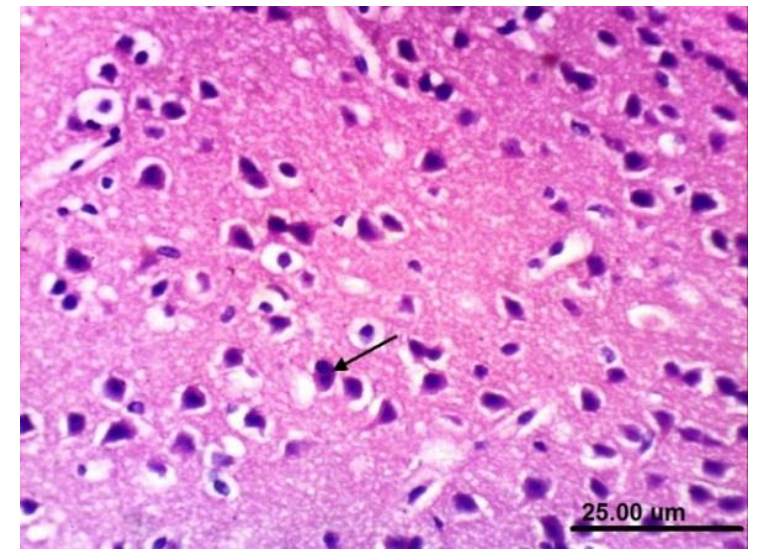

Figure 3: Brain of mice from $\mathrm{GVa}$ showing neuronophagia of degenerated neurons (arrow) ( $\mathrm{H} \&$ E, scale bar 25um).

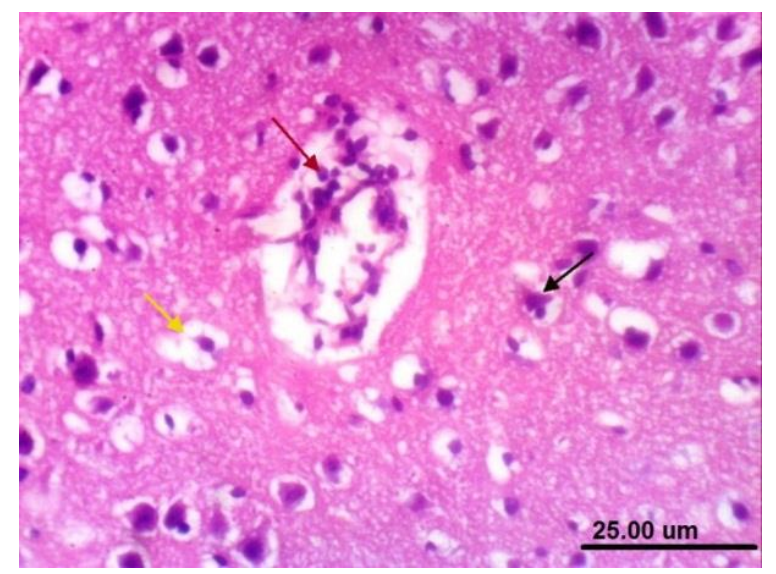

Figure 4: Brain of mice from group VII showing necrosis and pyknosis of neurons associated with neuronophagia ( black arrow), perivascular cuffing with mononuclear cells (red arrow) and intracellular vacuolation (yellow arrow). (H \& E, scale bar 25um).

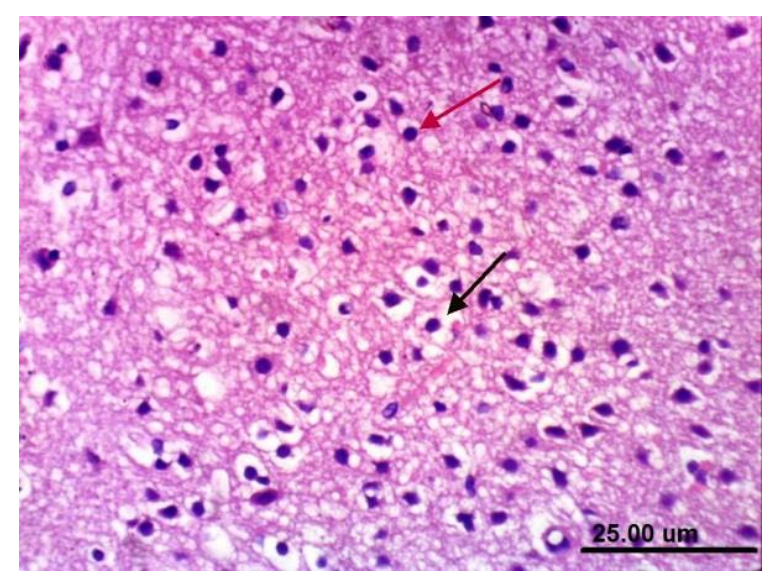

Figure 5: Brain of mice from GVa showing mild intracellular vacuolation and edema (black arrow) associated with proliferation of glia cells (red arrow)(H \& E, scale bar 25um).

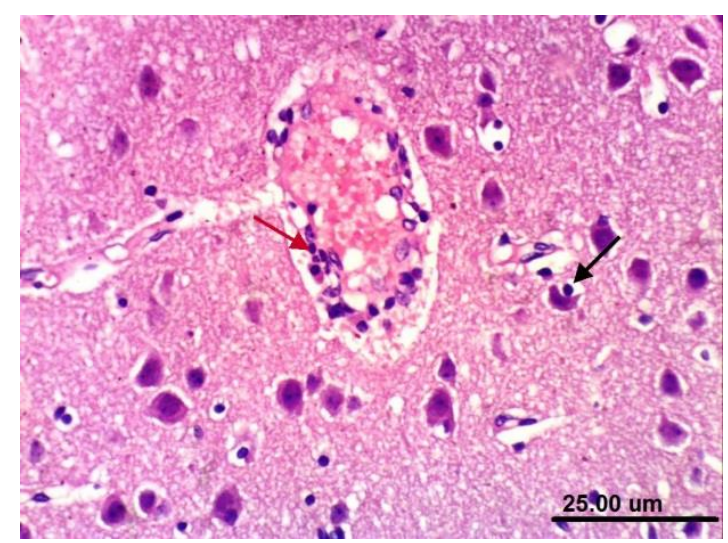

Figure 6: Brain of mice from group VII showing neuronophagia of necrotic neurons (black arrow)and mild perivascular cuffing with mononuclear cells (red arrow) (H \& E, scale bar 25um).

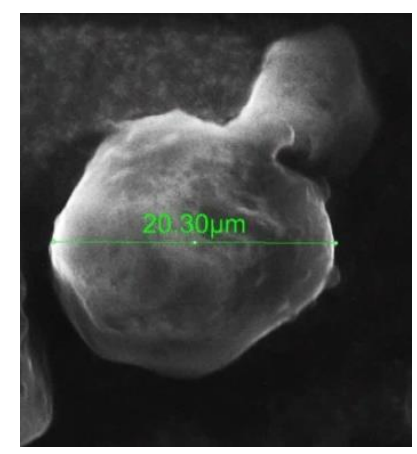

Figure 7: Brain tissue cyst of T.gondii from GII

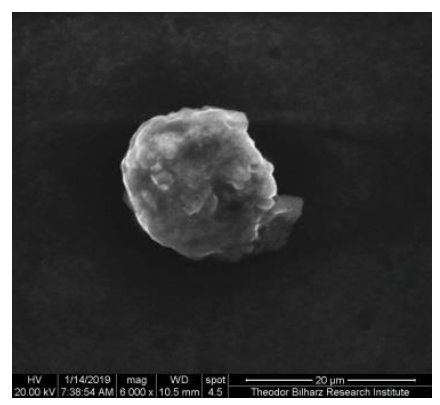

Figure 8: Brain tissue cyst of T.gondii from GIV.

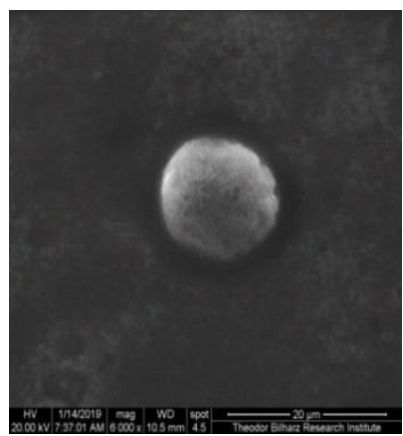

Figure 9: Brain tissue cyst of T.gondii from GVII 


\section{Discussion}

The aim of classification of the groups of mice into 2 subgroups is to observe the effect of tested drugs on brain tissue cysts either early or mature cysts.

As regards the parasitological results in chronic infection, the mean number of $T$. gondii brain tissue cysts was significantly decreased in all treated subgroups.

In the present study nitazoxanide significantly reduced number of brain tissue cysts which was in agreement with a previous study [14] that evaluated effect of nitazoxanide in acute and chronic toxoplasmosis.

A previous study [14] reported that NTZ significantly reduced the number of brain cysts in mice treated with it, explaining this effect as NTZ being an efficient immunomodulant. NTZ significantly increased IFN- $\gamma$ and enhanced the production of the inducible isoform nitric oxide synthases (iNOS) in brain tissue.

Higher iNOS production in brain tissues of NTZ treated mice was associated with lower parasitism. A previous study [15] found that low or even no iNOS production contributed to uncontrollable parasite multiplication, which supporting this mechanism of action of NTZ. A previous study analyzed the effect of NTZ and pyrimethamine on astrocytes infected with $T$. gondii in vitro and reported that NTZ reduced T. gondii infection more efficiently than pyrimethamine; additionally, NTZ was not cytotoxic to astrocytes at the administered dose [16].

Another mechanism that may contribute to parasite reduction was showed in experiments done by [17] who concluded that tizoxanide (TZX), the main active metabolite of NTZ which was not detectable in cerebrospinal fluid, can cross the BBB and distribute into the brain tissue. TZX is retained in brain much longer than that in plasma, which makes the pharmacokinetics of TZX in brain and plasma quite different.

Concerning nitazoxanide results, the present study also agreed with [8] who reported that using nitazoxanide reduces brain tissue cysts in chronically infected mice. Loading of nitazoxanide on silver nanoparticles increased its efficacy and significantly reduced brain tissue cysts which may be due to increasing the bioavailability and release of nitazoxanide into tissue after loading. This agreed with [18] who concluded that nanoparticles can act as drug carriers that can modulate pharmacokinetics, increase bioavailability and target release with minimal toxic effects. Another explanation that can clarify these results is the effect of AgNPs. A previous study [19] stated that AgNPs are potent 
enhancer to the immune system. They have significant adjuvant effect and they elicit both Th1 and Th2based immune responses. Also, they recruit leukocytes and increase cytokines levels (TNF-and IFN- $\gamma$ ).

This synergistic action between AgNPs and action of nitazoxanide in chronic infection may contribute to make this combination efficiently reducing tissue cysts in brain. In this study spiramycin slightly reduced brain tissue cyst count (significant reduction in comparison with infected control group), but when combined with nitazoxanide it was leading to detectable reduction in brain tissue cyst count. This may be due to spiramycin is unable to reach effective concentrations in the brain due to the presence of the efflux transporters multidrug resistant protein 2 and P-glycoprotein, however; spiramycin uptake in the brain can be augmented by co-administration of a drug that inactivate the efflux pump as antiparasitic metronidazole[20]. Similarly, [20] reported a significant reduction in the number of brain cysts after treatment with spiramycin-metronidazole in a mouse model of chronic toxoplasmosis. This combination of spiramycin and metronidazole which is anti-parasitic may explain effect of combination between spiramycin and nitazoxanide.

Combination of nitazoxanide loaded on silver nanoparticles and spiramycin accomplished the best therapeutic efficacy in treating chronic toxoplasmosis infections among the studied groups which may be due to their effectiveness as well as their synergistic action.

These result may be consistent with a previous study done [21] which reported that using Ag NPs, chitosan nanoparticles and spiramycin as a single or combined therapeutic agents against experimental toxoplasmosis, showed the highest degree of effectiveness attained by the synergistic action of these compounds as indicated by lower parasite count and higher IFN- $\gamma$, TNF$\alpha$ concentration.

Another parameter evaluating the present study was histopathological examination of brain sections by haematoxylin and eosin stain. As regards infected non treated control group, our results were in agreement with others [8] who noticed severe inflammatory reaction mainly mononuclear cells, congestion of meninges with mononuclear cells invading the meninges and necrosis of neurons. Toxoplasma cysts were found in meninges and embedded in the cerebral tissue.

Marked improvement in histopathological changes was detected in groups treated with nitazoxanide loaded silver nanoparticles in 2 subgroups and in which there was detectable improvement revealed by moderate inflammatory reaction exhibited by 
moderate intracellular vaculation, edema and mild perivascular cuffing with mononuclear cells. This was in agreement with another study [14] which reported that NTZ was significantly able to reduce inflammation and inflammatory lesions caused by $T$. gondii infection. Additionally, the highest dose of NTZ resulted in less pronounced inflammation and few infiltrating mononuclear cells. This reduction was expected due to the effective reduction in brain tissue cyst count which agrees with our results.

A previous study [22] investigated to gain further evidence for the anti-inflammatory properties of AgNPs, in which they used both in vivo and in vitro models and found that AgNPs are able to down regulate the quantities of inflammatory markers, suggesting that AgNPs could suppress inflammatory process.

The best improvement in histopathological picture was recorded in groups of mice that received combination of nitazoxanide loaded nanoparticles and spiramycin in two subgroups which may be attributed to the synergistic action between nitazoxanide loaded nanoparticles and spiramycin, that was in agreement with [21]

Confirming parasitological and histopathological results, SEM of T. gondii cysts from brain tissues of three groups in the subgroups of mice infected and treated 6weeks post infection was done.

The best result was to SEM of $T$. gondii cysts from brain tissues of the groups infected and treated with combination of nitazoxanide loaded nanosilver and spiramycin which showed shrinkage in their size, Its surface showed loss of normal contour in addition to formation of dimples compared to normal picture observed in the infected control group.

This may be attributed to AgNPs can be stored inside mitochondria, thus hampering the synthesis of adenosine triphosphate (ATP) [23] as well as causing damages to the mitochondrial membrane[24].

\section{Conclusion}

Nitazoxanide loaded on silver nanoparticles exhibit a significant inhibitory effect on chronically infected mice. Starting treatment early 2 weeks post infection may contribute in reduction in $T$. gondii brain tissue cysts. Loading of nitazoxanide on silver nanoparticles increases its approved potent therapeutic effect against experimental chronic toxoplasmosis.

\section{References}

1 Oz HS. "Fetal and maternal toxoplasmosis," Recent Advances in Toxoplasmosis Research, $1^{\text {st } E d n, ~}$ Chap.1, ed. C.M. Lee (Lexington, KY: Nova Science Publication) 2014; 1:1-33. 
2 Ali S, Mumar S, Kalam K, Raja K, Baqi S. Prevalence, clinical presentation and treatment outcome of cryptosporidiosis in immunocompetent adult patients presenting with acute diarrhoea. J Pak Med Assoc 2014; 64:613-618.

3 Edmundson MC, Capeness M, Horsfall L. Exploring the potential of metallic nanoparticles within synthetic biology. New Biotechnol 2014; $31: 572-578$.

4 Djurković-Djaković O, Milenkovic V, Nikolic A, Bobić B, Grujić J. Efficacy of atovaquone combined with clindamycin against murine infection with a cystogenic (Me49) strain of Toxoplasma gondii. J Antimicrob Chemother 2002; 50: 981-987.

5 Küünal S, Kutti S, Guha M, Rauwel P, Wragg D, Nurk G. Silver Nanoparticles Study for Application in Green Housing. ECS Trans 2015 ; 64: 15-24.

6 Prasad SR, Elango K, Damayanthi D, Saranya JS. Formulation and evaluation of azathioprine loaded silver nanopartilces for the treatment of rheumatoid arthritis. AJBPS 2013; 3:28-32.

7 Grujić J, Djurkovic-Djakovic O, Nikilic A, Klun I, Bobic D. Effectiveness of spiramycin in murine models of acute and chronic toxoplasmosis. Int $\mathbf{J}$ Antimicrob Agents 2005; 25: 226 -230.

8 Saleh MH, Abdel Maaboud AI, Selem RF, Elfadaly HA, Soliman NA. Therapeutic effect of nitazoxanide and zingiber officinale in treatment of chronic toxoplasmosis gondii using murine expermintal model. J Med Sci 2016; 37:207 227.

9 Piao MJ, Kang KA, Lee IK, Kim HS, Kim S, Choi JY. Silver nanoparticles induce oxidative cell damage in human liver cells through inhibition of reduced glutathione and induction of mitochondria-involved apoptosis. Toxicology Letters 2011; 201:92-100.

10 Huskinson J, Remington JS. Remarkable In Vitro and In Vivo Activities of the Hydroxynaphthoquinone 566C80 against Tachyzoites and Tissue Cysts of Toxoplasma gondii. Antimicrobial agents and chemotherapy 1991; 35: 293-299.

11 Suvarna SK, Layton C, Bancroft JD. Bancroft's Theory and Practice of Histological Techniques. Churchill Livingstone Elsevier, Oxford. 8th Edition, 2018; 672.

12 Dantas-Leite L, Urbina JA, De Souza W, Vommaro RC. Antiproliferative synergism of azasterols and antifolates against Toxoplasma gondii. International Journal of Antimicrobial Agents 2005; $25:$ 130-135.

13 Sawyer S. Analysis of Variance: The Fundamental Concepts. The Journal of manual \& manipulative therapy2009; 17(2):27-38.

14 El-Kowrany SI, Abd El Ghaffar AE, Shoheib ZS, Mady RF, Gamea GA. Evaluation of nitazoxanide as a novel drug for the treatment of acute and chronic toxoplasmosis. Acta.Tropica 2019; 195: 145-154.

15 Silva NM, Vieira JC, Carneiro CM, Tafuri WL. Toxoplasma gondii: the role of IFN-gamma, TNFRp55 and iNOS in inflammatory changes during infection. Exp Parasitol 2009; 123: $65-72$.

16 Galván-Ramírez MD, Dueñas Jiménez JM, Rocío L, Troyo-Sanroman R, Ramírez-Herrera M. Effect of nitaxozanide and pyrimethamine on astrocytes infected by Toxoplasma gondii in vitro. Arch Med Res 2013; 44: 415-421. 
17 Guo S, Li F, Wang B, Zhao Y, Wang X, Wei H. Analysis of tizoxanide, active metabolite of nitazoxanide, in rat brain tissue and plasma by UHPLC-MS/MS. Biomedical Chromatography 2019; 34: 1-7.

18 Khalil NM, de Mattos AC, Carraro TC, Ludwig DB, Mainardes RM. Nanotechnological strategies for the treatment of neglected diseases. Curr Pharm Des 2013; 19:73167329.

19 Xu Y, Tang H, Liu JH, Wang H, Liu Y. Evaluation of the adjuvant effect of silver nanoparticles both in vitro and in vivo. Toxicol Lett $2013 ; 219$ : 42-48.

20 Chew WK, Segarra I, Ambu SJ, Mak W. Significant reduction of brain cysts caused by Toxoplasma gondii after treatment with spiramycin co administered with metronidazole in a mouse model of chronic toxoplasmosis. Antimicrob. Agents Chemother 2012; 56: 1762-1768.
21 Hamad HK, Ramadan NF, Mohamed SH, Aly I, Zalat R. Study the synergistic Effect between Nanoparticles and Spiramycin on Immunological Response Against Toxoplasmosis. IOP Conf. Series: Materials Science and Engineering 2020;736: 1-9.

22 Wong CK, Cheung PF, Ip WK, Lam CW. Intracellular signaling mechanisms regulating toll-like Receptor mediated activation of eosinophils. Am J Respir Cell Mol Biol 2007; 37: 85-96.

23 Zheng J, Wu X, Wang M, Ran D, Xu W, Yang J. Study on the interaction between silver nanoparticles and nucleic acids in the presence of cetyl-trimethylammonium bromide and its analytical application. Talanta 2008; 74:526-532.

24 Adeyemi OS, Murata Y, Sugi T, Kato K. Inorganic nanoparticles kill Toxoplasma gondii via changes in redox status and mitochondrial membrane potential. Int J Nanomedicine 2017; 12: $1647-1661$.

To cite this article: Mohammed H. Saleh, Ibrahim M. Nagaty, Rabab S. Zalat, kawkab A. Ahmed, Doaa I. Yaseen, Reda M. Abdelhameed, Shereen M. Kishik. Assessment of Nitazoxanide Loaded on Silver nanoparticles Efficacy on Treatment of Murine Model of Chronic Toxoplasmosis. BMFJ 2021; 38 (academic issue): 186-199. DOI: $10.21608 / \mathrm{bmfi} .2021 .58655 .1372$ 